


\title{
Nonequilibrium Nematic-Isotropic Interface
}

\author{
Oscar Nassif de Mesquita \\ Departamento de Física \\ ICEX, Universidade Federal de Minas Gerais \\ C.P. 702, Belo Horizonte 30161-960, MG
}

Received 28 October, 1998

\begin{abstract}
Liquid crystals have been very fruitful systems to study equilibrium phase transitions. Recently, they have become an important system to study dynamics of first-order phase transitions. The moving nonequilibrium nematic-isotropic interface is a model system to study growth of stable states into metastable states and displays a myriad of dynamical instabilities that, far from equilibrium, drive the system to a scenario of spatio-temporal chaos. We present a mean-field theory for the time evolution of a planar nonequilibrium nematicisotropic interface for pure liquid crystals using a time dependent Ginzburg-Landau equation, which is one of the simplest approaches to dissipative dynamics. We obtain a theoretical expression for the growth kinetics of the nematic phase into a metastable isotropic phase and compare it with our experimental results. In a directional solidification arrangement we study instabilities of the nematic-isotropic interface of the liquid crystal $8 \mathrm{CB}$ doped with water and hexachloroethane. The observed instabilities are similar to cellular instabilities that appear during growth of crystal-melt interfaces of binary mixtures. We then compare our results with known theories of morphological instabilities during crystal growth.
\end{abstract}

\section{Introduction}

Equilibrium thermodynamics has contributed much to the understanding of a variety of physical phenomena. Like other areas of Physics, equilibrium thermodynamics is based on some variational principle (maximum entropy or minimum free-energy), such that if we know the free-energy of the system then we are able to predict all the properties of its, in general, unique equilibrium state.

The diversity of shapes, forms (including living forms) in Nature, certainly could not be explained by equilibrium thermodynamics. We do not know if nonequilibrium thermodynamics can explain all that either, but at least it opens up some new possibilities. In nonequilibrium thermodynamics, history (time evolution) assumes its importance leading the system to a variety of different states, other than equilibrium states, as energy is continuously pumped into it. When the input of energy ceases, the system then evolves to its equilibrium state - that is the boring state of death for living forms. On the other hand, nonequilibrium systems display a variety of rich and complex dynamical phenomena, self-organization and pattern formation giving rise to a great diversity of forms.

As far as I know, there is nothing like a general variational principle for nonequilibrium thermodynamics. We cannot say in general that the time evolution or steady-states of nonequilibrium systems are determined by some principle like minimum dissipation of energy, or other like maximum production of entropy. In some especial cases these principles work and in others they do not work, raising some doubts about the usefulness of such general principles. In hydrodynamic systems one can find both situations [1] [2]. Therefore, the usual approach to study nonequilibrium systems is to consider each case and look for some universal behavior. Here, universal behavior means that the time evolution and steady-states of several different real systems can be grouped and described by solutions of a particular, usually phenomenological, nonlinear partial differential equation [2].

In the present work we will discuss two different sit- 
uations, both with very rich dynamical behavior. In one case we shall discuss, for pure liquid crystals, the motion of a planar nematic-isotropic interface when the temperature of the system is quenched below its equilibrium nematic-isotropic transition temperature, such that the nematic phase (stable phase) grows into the isotropic phase (metastable phase). Which orderparameter-shape-profile and front velocity will be selected out from several possible solutions of nonlinear equations is what we would like to determine. One of the simplest equations for dissipative dynamics is a time-dependent Ginzburg-Landau equation [3 ] [4] [5 ]. We will derive the kinetics of the nematic-isotropic interface based on this equation, discuss the velocityselection problem and then compare the results with our kinetic measurements [6] [7 ].

In the second situation, we will describe morphological instabilities that occur in the moving nematicisotropic interface of doped systems using an experimental configuration of directional solidification. These instabilities (cellular and dendritic) have been studied for more than 30 years [8] [9] [10 ] in the context of crystal-growth, because they are very important for material processing. They are the main responsible for the microstructure of cast metals, and, consequently, responsible for their mechanical and electrical properties. More recently, the cellular instability was shown to occur also in liquid crystals [11] [12] [13], displaying a variety of new dynamical phenomena and a scenario of spatio-temporal chaos [14 ] [15 ]. Since for liquid crystals the parameters for triggering the cellular instability and time scales involved are more amenable to laboratory conditions, the moving nematic-isotropic interface has become a model system to study complex dynamics and pattern formation. We will discuss the physical origin for the onset of the cellular instability and show some of our more recent data for the moving nematic-isotropic interface of the liquid crystal $8 \mathrm{CB}$ doped with hexachloroethane and water.

\section{Nematic-isotropic phase tran- sition}

\section{A. Pure systems}

The nematic-isotropic phase transition in liquid crystals is a weakly first-order transition. In the nematic phase the rodlike anisotropic molecules interact to create a long-range orientational order. The molecules are aligned, in the average, along a particular direction defined by a vector $\vec{n}$, the director. In the nematic phase the center of mass of the molecules are not correlated, therefore, concerning the translation degrees of freedom the liquid crystal behaves as a liquid : an anisotropic liquid. The quadrupolar ordering of the nematic phase can be described by a traceless tensor, the order parameter tensor, given by [16 ]

$$
Q_{\alpha \beta}=\frac{Q}{2}\left(3 n_{\alpha} n_{\beta}-\delta_{\alpha \beta}\right)
$$

where $n_{\alpha}$ are the components of the director vector $\vec{n}$ and $Q(0 \leq Q \leq 1)$ is the modulus of the order parameter. As seen in other articles of this issue, the Landaude Gennes theory [16 ] can provide a phenomenological mean-field description of phase transitions in liquid crystals. The Landau-de Gennes free energy density for nematic liquid crystals can be written as,

$$
F=F_{0}+\frac{1}{2} C_{1} Q_{\alpha \beta} Q_{\beta \alpha}+\frac{1}{3} C_{2} Q_{\alpha \beta} Q_{\beta \gamma} Q_{\gamma \alpha}+\ldots
$$

where $F_{0}$ is the free energy density of the isotropic phase and $C_{1}, C_{2}$, etc., are constants.

In our experiments, the liquid crystal $8 \mathrm{CB}$ is sandwiched between glass slides. Typical sample thicknesses vary from 3 to $5 \mu \mathrm{m}$. The internal surfaces of the cover glasses which are in contact with the liquid crystal are treated with silane, what guarantees a homeotropic configuration for the nematic phase, i.e., the director $\vec{n}$ is perpendicular to the cover glass surfaces. In this simplified geometry, where $Q_{z z}=Q, Q_{y y}=Q_{x x}=-Q / 2$ $Q_{x y}=Q_{y z}=Q_{x z}=0$, the Landau-de Gennes free energy density can be written in terms of the scalar order parameter $Q$ as,

$$
F=\frac{1}{2} A Q^{2}-\frac{1}{3} B Q^{3}+\frac{1}{4} C Q^{4}+\frac{1}{2} a \xi_{0}^{2} T^{*}\left(\frac{\partial Q}{\partial z}\right)^{2}
$$

where $A, B, C$ and $\xi_{0}$ are phenomenological coefficients whose values must be determined empirically from experiments. As usual, one assumes that the main temperature dependence is contained in $A$ such that $A=$ $a\left(T-T^{*}\right)$, where $T$ is temperature, $T^{*}$ is the temperature at which a second-order phase transition would 
occur if $\mathrm{B}=0$ exactly, and $a$ is a constant. The coefficient of the gradient term is also a constant which we wrote as $a \xi_{0}^{2} T^{*}$, anticipating future results. $\xi_{0}$ is the bare correlation length with dimension of the order of the molecular size. Without losing generality we set the free energy $F_{0}$ of the isotropic phase equal to zero. A schematic view of this free energy as a function of the order parameter for different temperatures and without the gradient term, can be seen in Fig.1. $\mathrm{T}_{N I}$ is the nematic-isotropic transition temperature and $\mathrm{Q}_{N I}$ is the order parameter at the transition. The free energy without the gradient term can describe homogeneous phases. In order to describe the nematic-isotropic interface we have to include it. Let us use the homogeneous free energy (no gradient term) to make some predictions for the homogeneous phases.

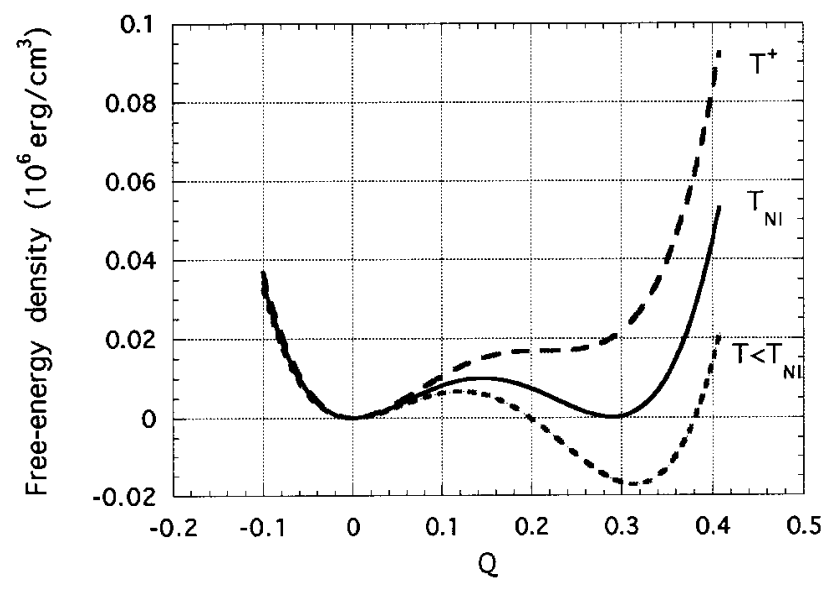

Figure 1. Plot of Landau-de Gennes homogeneous free energy density $F$ for the nematic-isotropic phase transition as a function of the scalar order parameter $Q$, for different temperatures. Parameters used are for the nematic-isotropic transition of the liquid crystal $8 \mathrm{CB}$.

To find the conditions describing a first-order phase transition we shall, as usual, minimize the free energy density and impose the coexistence between the isotropic and nematic phases, i.e., $\partial F / \partial Q=0$ and $F\left(Q_{N I}\right)=F(0)=0$, where $Q_{N I}$ is the value of the order parameter of the nematic phase at the transition temperature $T_{N I}$ and $Q=0$ is the value of the order parameter of the isotropic phase. Note that the temperature $T_{N I}$ is different from the phantom second-order transition temperature $T^{*}$. By imposing the conditions above we find:

$$
T_{N I}-T^{*}=\frac{2}{9} \frac{B^{2}}{a C}
$$

$$
Q_{N I}=\frac{2}{3} \frac{B}{C} .
$$

From Fig.1 we see that for $T>T_{N I}$, the nematic phase $(Q \neq 0)$ has a local minimum (metastable phase) while the isotropic phase $(Q=0)$ has an absolute minimum (stable phase). For $T<T_{N I}$ the opposite occurs: the nematic phase is the stable one while the isotropic is the metastable phase. At $T=T^{*}, \partial^{2} F / \partial Q^{2}=0$, for $Q=0$, therefore the isotropic phase becomes absolutely unstable. We also define the temperature $T^{+}$, given by $T^{+}-T_{N I}=\frac{1}{8}\left(T_{N I}-T^{*}\right)$, where $\partial^{2} F / \partial Q^{2}=0$, for $Q^{+}=\frac{3}{4} Q_{N I}$, such that the nematic phase becomes absolutely unstable. Therefore, the metastable region occurs for $T^{*} \leq T \leq T^{+}$and this is the temperature interval where a nematic-isotropic interface can exist. A fundamental question that must be answered is how fast one phase grows at the expenses of the other, when the system is quenched from one temperature to another. This question is not trivial to answer and great efforts have been put into it in the last decades [3 ] [4 ] [5]. This is the sort of question that we would like to answer when we consider a nonequilibrium nematicisotropic interface.

\section{Nematic-isotropic interface}

At $T_{N I}$ the nematic and isotropic phases can coexist, separated by a stationary interface. If we consider the complete free energy density given by Eq. (3) and imposing the boundary conditions $Q(z=+\infty)=0$ and $Q(z=-\infty)=Q_{N I}$, we can find the profile for $Q(z)$. To minimize the free energy density $(\delta F / \delta Q=0)$ we then solve the Euler-Lagrange equation associated with Eq. (3). The resulting equation is

$$
a \xi_{0}^{2} T^{*} \frac{\partial^{2} Q}{\partial z^{2}}=A Q-B Q^{2}+C Q^{3}
$$

with solution

$$
Q(z)=\frac{Q_{N I}}{2}\left[1-\tanh \left(\frac{z}{2 \xi}\right)\right]
$$

where the correlation length $\xi$ is related to the bare correlation length $\xi_{0}$ by

$$
\xi=\xi_{0} \sqrt{\frac{T^{*}}{T-T^{*}}}
$$


The interface is located at $z=0$ and its width is determined by $\xi$. The equilibrium nematic-isotropic surface tension can be calculated using the excess surface free energy definition [17 ] [5 ]

$$
\gamma_{e}=\int_{-\infty}^{+\infty} \frac{1}{2} A_{e} \xi_{e}^{2}\left(\frac{\partial Q}{\partial z}\right)^{2} d z=\frac{1}{6} A_{e} \xi_{e} Q_{N I}^{2}
$$

where all the quantities are calculated at $T_{N I}$.

\section{Nonequilibrium nematic-isotropic interface}

If we prepare the system initially at the equilibrium temperature $T_{N I}$, with boundary conditions $Q(z=$ $+\infty)=0$ and $Q(z=-\infty)=Q_{N I}$, we will have the profile $Q(z)$ given by Eq. (7). Then we quench the temperature to a value smaller than $T_{N I}$ but larger than $T^{*}$. The interface will start to move towards positive $z$, i.e., the nematic phase starts to grow at the expenses of the isotropic phase. It has been proposed in the literature some theoretical frameworks to compute this velocity as a function of temperature. We will describe below the simplest one for dissipative dynamics based on a time dependent Ginzburg-Landau equation, given by [3] [4] [5] [18]

$$
\eta \frac{\partial Q}{\partial t}=-\frac{\delta F}{\delta Q}
$$

where $\eta$ is a viscosity associated with the ordering of the nematic phase. A transport parameter that can be measured directly is the rotational viscosity of the nematic liquid crystal $\gamma_{1}$, which is related to $\eta$ by [5] [18 ]

$$
\eta=\frac{\gamma_{1}}{3 Q_{N I}^{2}}
$$

The equation we have to solve is then,

$$
\eta \frac{\partial Q}{\partial t}=a \xi_{0}^{2} T^{*} \frac{\partial^{2} Q}{\partial z^{2}}-A Q+B Q^{2}-C Q^{3}
$$

We look for stationary solutions moving with constant speed like $Q(z-V t)$. By changing variable, $s=z-V t$, we obtain,

$$
a \xi_{0}^{2} T^{*} \frac{d^{2} Q}{d s^{2}}+\eta V \frac{d Q}{d s}-A Q+B Q^{2}-C Q^{3}=0
$$

We can try a solution of the type,

$$
Q(s)=\frac{\alpha}{2}\left[1-\tanh \left(\frac{s}{2 \Delta}\right)\right]
$$

where $\alpha$ and $\Delta$ are to be determined. We will show that this is indeed a solution of Eq. (13). This is not, however, the only solution. The velocity $V$ depends on the shape of the interface. We can find a great number of shape solutions each one moving with different speed. Ben Jacob et al. and later W. van Saarlos [19 ] [4], made a nonlinear marginal stability analysis of propagation of fronts into metastable states described by an equation of the type of Eq. (13). They showed that, within the metastable range, the only selected velocity is the one of a moving front with profile defined by Eq. (14). Then we can use this profile and uniquely determine the velocity of the front.

We substitute Eq. (14) into Eq. (13) and obtain,

$$
\begin{gathered}
\alpha=\frac{3}{4} Q_{N I}\left[1+\sqrt{1-\frac{8}{9} \frac{\left(T-T^{*}\right)}{\left(T_{N I}-T^{*}\right)}}\right] \\
\Delta=\frac{4}{3} \frac{\xi_{e}}{\left[1+\sqrt{1-\frac{8}{9} \frac{\left(T-T^{*}\right)}{\left(T_{N I}-T^{*}\right)}}\right]}
\end{gathered}
$$

and finally, the expression for the front velocity is

$$
V=\frac{3}{4} \frac{a \xi_{e}}{\eta}\left(T_{N I}-T^{*}\right)\left[\sqrt{1-8\left(\frac{T-T_{N I}}{T_{N I}-T^{*}}\right)}-1\right]
$$

If $T<T_{N I}$ the interface moves towards positive $\mathrm{z}$ and it is supercooled. If $T>T_{N I}$ the interface moves towards negative $\mathrm{z}$ and it is superheated. The difference $\Delta T=T-T_{N I}$ is called supercooling or superheating depending on its sign. From Eq. (15), for temperatures above $T^{+}$, the front velocity becomes imaginary: the nematic phase is absolutely unstable. For the liquid crystal $8 \mathrm{CB}, T_{N I}-T^{*}$ is equal to $2 \mathrm{~K}$, therefore $T^{+}-T_{N I}$ is equal to $0.25 \mathrm{~K}$. In fact we observe, even for small superheatings like $0.3 \mathrm{~K}$, that the interface disappears and the transition resembles a second order transition.

For a first order transition and very small supercoolings or superheatings the velocity of the front should vary linearly with $\Delta T$. If we expand Eq. (15) imposing that $\Delta T=T-T_{N I}<<\left(T_{N I}-T^{*}\right) / 8$ we obtain,

$$
V=\frac{3 a \xi_{e}}{\eta}\left(T_{N I}-T\right)
$$

This linear behavior is difficult to observe in the case of our liquid crystal $8 \mathrm{CB}$ because the linear region occurs for undercoolings or superheatings much smaller 
than $0.25 \mathrm{~K}$. This short temperature interval for the linear behavior is a characteristic of weakly first-order transitions. For instance, in the case of crystal-melt interfaces this linear behavior can extend to larger temperature intervals. We will fit our data of velocity as a function of temperature using the complete Eq. (15).

In the above analysis we have neglected the latent heat liberated during the motion of the interface. In the case of the nematic-isotropic transition of the liquid crystal $8 \mathrm{CB}$ the latent heat generated is very small and can be safely neglected. This is one of the advantages of studying kinetics of nematic-isotropic interfaces rather than crystal-melt interfaces. In addition, for the nematic-isotropic interface the Landau-de Gennes free energy formulation can give reasonable results and then we can make predictions based on this free energy, like the velocity of the front shown above. For regular crystal-fluid interfaces, as far as I know, there is no such free energy description for the problem.

\section{B. Doped systems}

If we introduce dopants that are soluble or partially soluble into the liquid crystal, a binary phase will emerge. For very low concentrations a sketch of a binary phase diagram is shown in Fig. 2. There we show a situation where the dopant decreases the free energy of the solvent, then the transition temperature decreases as the concentration of dopant increases. Also, a region of coexistence of nematic and isotropic phases appears between the liquidus and solidus lines. The liquidus and solidus temperatures can be written as

$$
\begin{gathered}
T_{L}=T_{N I}-m_{L} C_{I} \\
T_{S}=T_{N I}-m_{S} C_{N},
\end{gathered}
$$

where $m_{L}$ and $m_{S}$ are the liquidus and solidus slopes and $C_{N}$ and $C_{I}$ are the dopant concentrations in the nematic and isotropic phases respectively. For a given temperature the dopant concentration in the nematic phase $\left(C_{N}\right)$ is in equilibrium with a dopant concentration in the isotropic phase $\left(C_{I}\right)$, such that the ratio $C_{N} / C_{I}=m_{L} / m_{S}=K$, where $\mathrm{K}$ is the segregation coefficient. In the present case $K<1$.

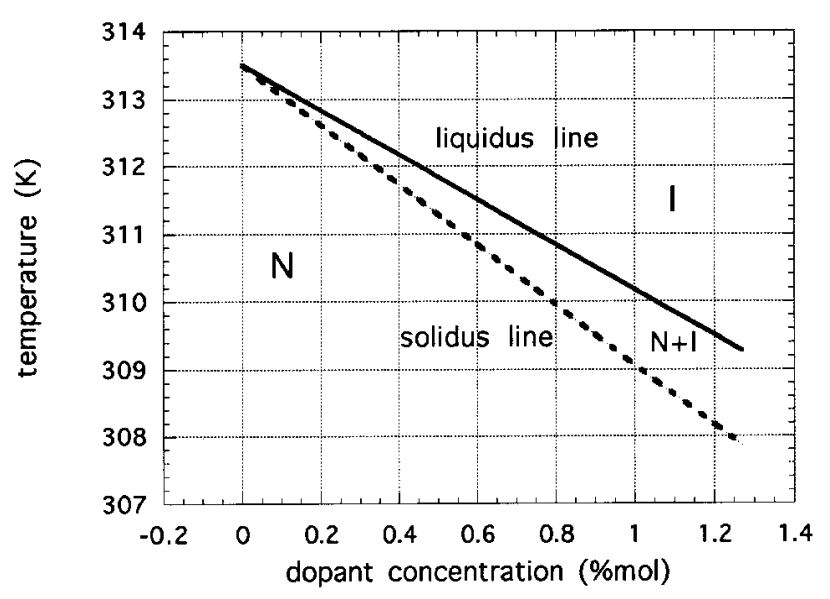

Figure 2. Partial plot of a phase diagram for a binary mixture with segregation coefficient $\mathrm{K}$ smaller than one. The transition temperature decreases linearly with increasing dopant concentration, for small dopants concentrations. Below the solidus line is the nematic phase $(\mathrm{N})$, above the liquidus line is the isotropic phase (I), between the two is a region of coexistence of the two phases $(\mathrm{N}+\mathrm{I})$. Parameters used are for water in $8 \mathrm{CB}$ with $K=0.75$ and $m_{L}=3.33$ $\mathrm{K} / \% \mathrm{~mol}$.

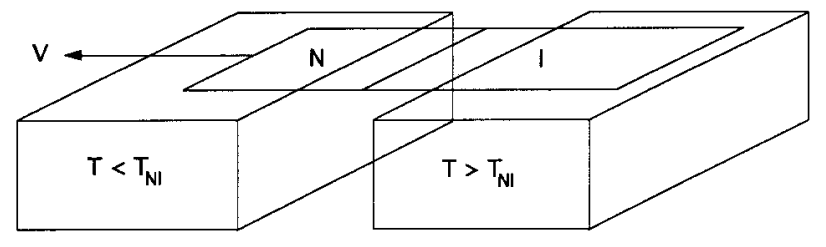

Figure 3. Directional solidification apparatus. A thin sample of liquid crystal sandwiched between glass slides is placed in contact with two aluminum blocks, one above and the other below the nematic-isotropic transition temperature, separated by a $1 \mathrm{~cm}$ glass gap. The nematic-isotropic interface is visualized in the gap with a microscope connected to a video-system. As the sample is pulled towards the colder block the nematic phase starts to grow. We can revert the pulling system and also melt the nematic phase. The motion of the interface is then video-recorded for posterior analysis.

Let us consider now a experimental situation where the liquid crystal is sandwiched between glass slides as mentioned before and put in a directional solidification oven like in Fig. 3. The oven consists of two metal blocks separated by some distance. One of the metal blocks has temperature above $T_{N I}$ and the other below, in such a way that a temperature gradient appears on the sample when it is placed in thermal contact with 
the two blocks. A nematic-isotropic interface will appear in the space between the two blocks. This interface can be visualized by an optical microscope coupled to a video system. A schematic plot of the equilibrium concentration of dopants is shown in Figure 4a. By using a pulling system we start to pull the sample towards the colder block, then the nematic phase starts to grow. We follow the motion of the interface until it becomes stationary in the laboratory frame. At this situation we know that the interface is moving with the same velocity as the pulling velocity. Since $K<1, C_{N}<C_{I}$, then as the nematic phase grows it segregates dopants at the interface, then the dopant concentration at the isotropic side of the interface starts to build up. The steady-state situation is achieved when the segregated dopant flux equals the dopant flux due to diffusion in both phases. The transport equations for the dopants in the system of reference of the moving interface are given by [9 ] [10 ],

$$
\begin{gathered}
\frac{\partial C_{N}}{\partial t}=D_{N} \frac{\partial^{2} C_{N}}{\partial z^{2}}+V \frac{\partial C_{N}}{\partial z} \quad(z<0) \\
\frac{\partial C_{I}}{\partial t}=D_{I} \frac{\partial^{2} C_{I}}{\partial z^{2}}+V \frac{\partial C_{I}}{\partial z} \quad(z>0)
\end{gathered}
$$

with boundary conditions,

$$
V C_{I}(1-K)=D_{N} \frac{\partial C_{N}}{\partial z}-D_{I} \frac{\partial C_{I}}{\partial z}
$$

at the interface

$$
\begin{gathered}
(z=0), \\
C_{N}(z=-\infty)=C_{I}(z=+\infty)=C_{0},
\end{gathered}
$$

where $D_{N}, D_{I}$, are the diffusion coefficients of the dopant in the nematic and isotropic phases respectively, $\mathrm{V}$ is the velocity of the interface and $C_{0}$ is the initial dopant concentration in the isotropic phase. The steady-state solution for this problem is given by,

$$
\begin{gathered}
C_{N}(z)=C_{0} \text { for } z<0 \\
C_{I}(z)=C_{0}\left[1+\frac{1-K}{K} \exp \left(-\frac{V}{D_{I}} z\right)\right] \text { for } z>0
\end{gathered}
$$

A steady-state dopant concentration profile given by Eq (19.b) is shown in Fig. 4b. The liquidus temperature ahead of the interface and the actual temperature profile in the sample is shown in Fig. 5. If the external temperature gradient is such that the actual temperature of the interface is smaller than the liquidus temperature (like in the case of Fig. 5), the liquid ahead of the interface is supercooled ("constitutional supercooling"). The interface is then growing into a metastable phase, then instabilities occur: the planar interface becomes cellular. This morphological instability is named Mullins-Sekerka instability [8] and has been the subject of intense research for the last 30 years [8 ] [9] [11 ] [15] [12 ] [13].
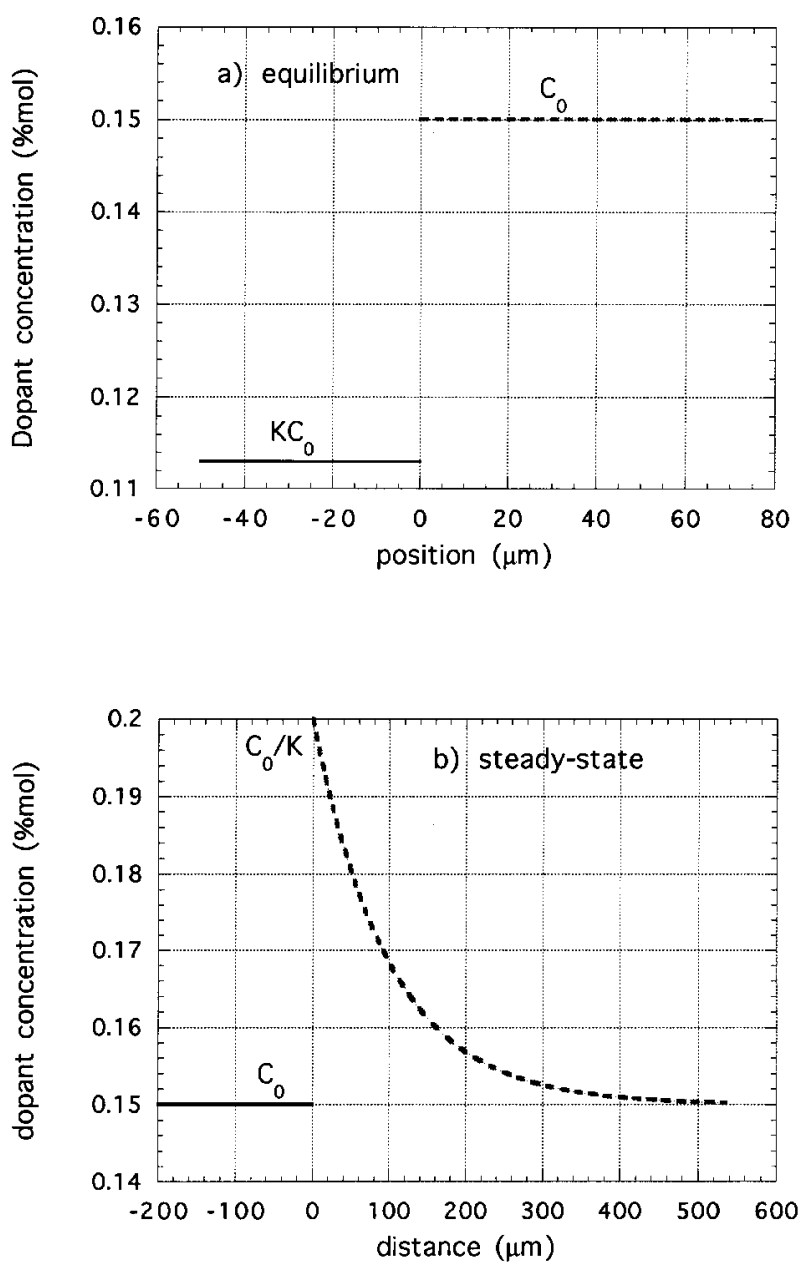

Figure 4. a) Equilibrium dopant concentration at the nematic-isotropic interface $(V=0)$. Parameters are for water in $8 \mathrm{CB}: K=0.75$ and dopant concentration in the isotropic phase equal to $C_{0}=0.15 \%$ (saturation concentration of water in the isotropic phase). Interface is at $z=0$, nematic phase is at $z<0$ and isotropic phase is at $z>0$; b) Steady-state dopant concentration profile at a moving nematic-isotropic interface with velocity $V=0.7 \mu \mathrm{m} / \mathrm{s}$, as predicted by Eq. (19.a) and Eq. (19.b). Interface is at $z=0$, nematic phase is at $z<0$ and isotropic phase is at $z>0$. Parameters used are for water in $8 \mathrm{CB}$ with $C_{0}=0.15 \%, K=0.75$ and $D_{I}=70 \mu \mathrm{m}^{2} / \mathrm{s}$. 


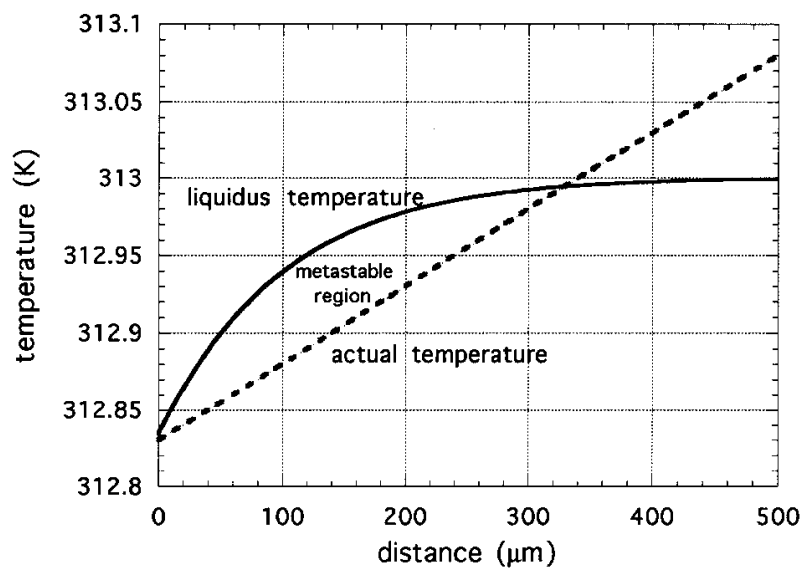

Figure 5. Continuous line is the liquidus temperature in the isotropic side of the interface for the steady-state dopant concentration profile of Fig. 4b, given by Eq. (17.a), with $T_{N I}=313.5 \mathrm{~K}$ and $m_{L}=3.33 \mathrm{~K} / \% \mathrm{~mol}$. The dashed line represents the external temperature profile determined by the temperatures of the ovens of the directional solidification apparatus. In the case shown here the temperature gradient $G=5 \mathrm{~K} / \mathrm{cm}$ such that, near the interface, the actual temperature of the isotropic phase is below the transition (liquidus) temperature (constitutional supercooling). The interface is then moving towards a metastable phase, then cellular instabilities can occur. By increasing the external temperature gradient, or decreasing the growth velocity this instability can be avoided.

We are interested on the weakly non-linear regime of such instabilities, where a simple phenomenological description is possible. If only few Fourier modes of the interface deformations are unstable and the rest is damped due to dissipation, we can describe the dynamics by a simple Landau amplitude equation of the type [2] [10 ],

$$
\frac{\partial A}{\partial t}=\omega A-\sigma|A|^{2} A
$$

where $A$ is the amplitude of the most unstable spatial Fourier mode, $\omega$ is the growth rate of this mode and $\sigma$ is the third-order coefficient. This is the simplest Landau equation allowed by the symmetry of the problem.

A solution to Eq. (20.a) is,

$$
|A|=\left[\sqrt{\left(\frac{\sigma}{\omega}+\left(\frac{1}{\left|A_{0}\right|^{2}}-\frac{\sigma}{\omega}\right) \exp (-2 \omega t)\right)}\right]^{-1} .
$$

where $A_{0}$ is the initial amplitude at $t=0$. The behavior above has been observed in hydrodynamic systems specially for the Rayleigh-Benard instability [2 ]. The first demonstration of this universal behavior in directional solidification was made by us [12 ], using a nonequilibrium nematic-isotropic interface. As the growth velocity is increased the system is taken far from equilibrium, secondary instabilities starts to occur and the interface can become chaotic. Coullet et al. [20 ] showed that the symmetry of the problem allows 10 different secondary instabilities. Some of them have been already observed [15 ] . Kassner et al. [14 ] have studied some of the scenarios of spatio-temporal chaos in this system.

We shall later present our data displaying some of these instabilities. The nonequilibrium nematicisotropic interface is a very rich system to study nonequilibrium dynamics and pattern formation.

\section{Experiments}

\section{A. Kinetic measurements for pure systems}

Kinetic measurements are done with pure liquid crystal $8 \mathrm{CB}$. The system initially in the isotropic phase at temperatures little above $T_{N I}$ is quenched down to temperatures slightly below $T_{N I}$. A moving nematicisotropic interface is then observed and recorded with video techniques. Velocity of the front is measured as a function of temperature. The apparatus oven is made with a large copper block and visualization of the interface is made with an optical microscope through sapphire windows. Sapphire windows are used because of their high heat conduction coefficient, such that the temperature profile in the sample is little affected by the ambient temperature and stays very uniform and stable. The temperature is monitored by a thermocouple inside of the copper block. Temperature stability is of $\pm 0.01 K$ during the time interval of each run. The relaxation time for thermal equilibrium after quenching the temperature is around $3 \mathrm{~s}$. We position the microscope at the opposite end of our $7 \mathrm{~cm}$ sample in relation to where the interface starts, such that the actual data is taken after the temperature has equilibrated. Clearly, we cannot safely measure very fast interfaces with high supercoolings, then we limit the measurements to interface velocities up to 5 to $6 \mathrm{~mm} / \mathrm{s}$, which will take about 10s to enter the field of view of the microscope, i.e., minimum of 3 times the thermal relaxation time.

A plot of velocity as a function of temperature is shown in Fig. 6 [6] [7]. The data are circles with associated error bars. The continuous line is a fit to 
an expression like Eq. (15), where we use the value $T_{N I}-T^{*}=2 K$ for $8 \mathrm{CB}$, such that

$$
V=\mu\left(\sqrt{1-4\left(T-T_{N I}\right)}-1\right),
$$

and with the help of Eq. (11) we get,

$$
\mu=\frac{3}{4} \frac{a \xi_{e}}{\eta}=\frac{9}{2} \frac{a \xi_{e} Q_{N I}^{2}}{\gamma_{1}}
$$

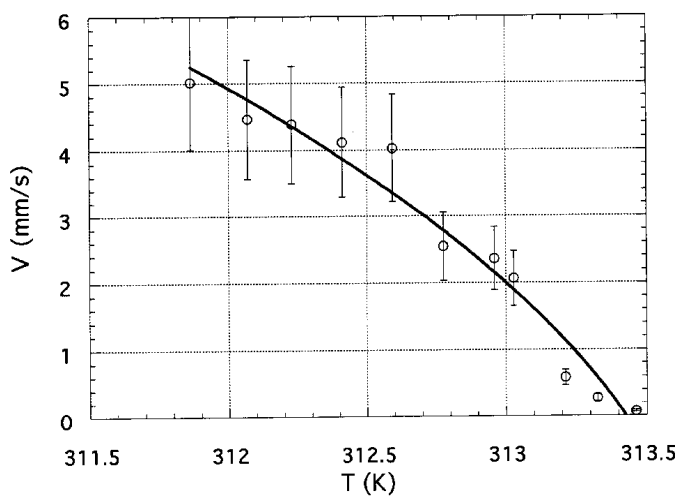

Figure 6. Plot of the velocity $V$ of the moving planar nematic-isotropic interface as a function of temperature $T$. The circles with error bars are the experimental data for the pure liquid crystal $8 \mathrm{CB}$. Continuous line is a fit to Eq. (15).

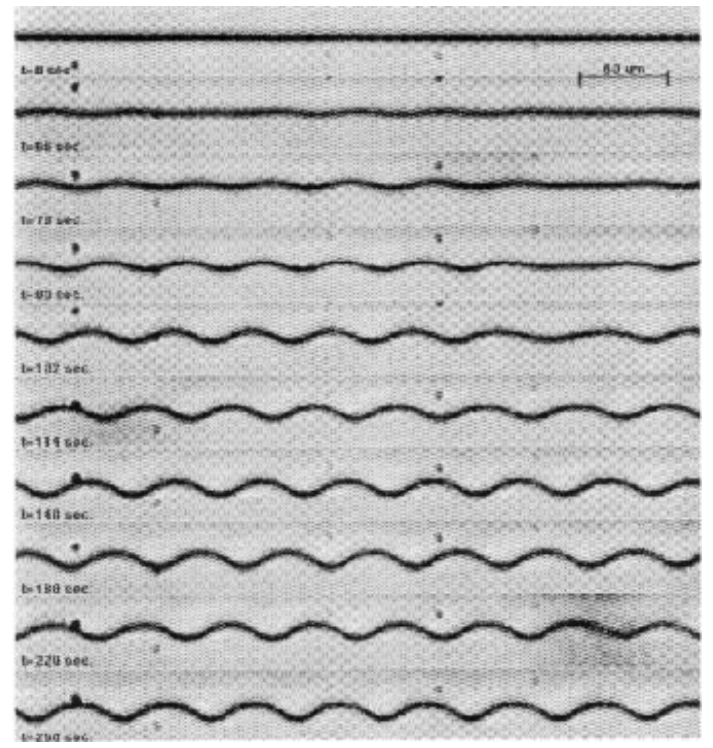

Figure 7. Time series of a doped moving nematic-isotropic interface undergoing a cellular instability.

From the fitting we obtain the value $\mu=3.1 \pm 0.5$ $\mathrm{mm} / \mathrm{sK}$. We have to compare this value with the theoretical prediction above. The measurements of the rotational viscosity $\gamma_{1}$ were made by Viana et al.[6 ] [7 ] , using the technique of optical birefringence. Near $T_{N I}$ its value is $\gamma_{1}=0.25 \pm 0.05$ poise. The values for the other parameters were measured by Faetti et al. [17 ] and are,

$$
\begin{aligned}
& a=(1.9 \pm 0.1) \cdot 10^{6} \mathrm{erg} / \mathrm{cm}^{3} \mathrm{~K} \\
& \xi_{e}=\left(18_{-10}^{+17}\right) \cdot 10^{-8} \mathrm{~cm} \\
& Q_{N I}=(0.29 \pm 0.01) .
\end{aligned}
$$

With these values we obtain

$\mu=5.2 \mathrm{~mm} / \mathrm{sK}$, with a lower limit of $2.3 \mathrm{~mm} / \mathrm{sK}$ and upper limit of $10.4 \mathrm{~mm} / \mathrm{sK}$. The largest error comes from the measurements of the correlation length. Our result falls within this range.

\section{B. Morphological instabilities in doped systems}

Our measurements of instabilities on the moving nematic-isotropic interface is done in a directional solidification apparatus described earlier and presented in Fig. 3. The sample consists of the liquid crystal $8 \mathrm{CB}$ doped with $1 \% \mathrm{~mol}$ of hexachloroethane $\mathrm{C}_{2} \mathrm{Cl}_{6}$, or with $0.15 \%$ mol of water. In this paper we just want to give a flavor about the possibilities and wealth of dynamical phenomena that the nonequilibrium nematic-isotropic interface displays. For a more complete description of experiments made by us and novel results with applied electric field see references [12 ] [13 ] and [21 ].

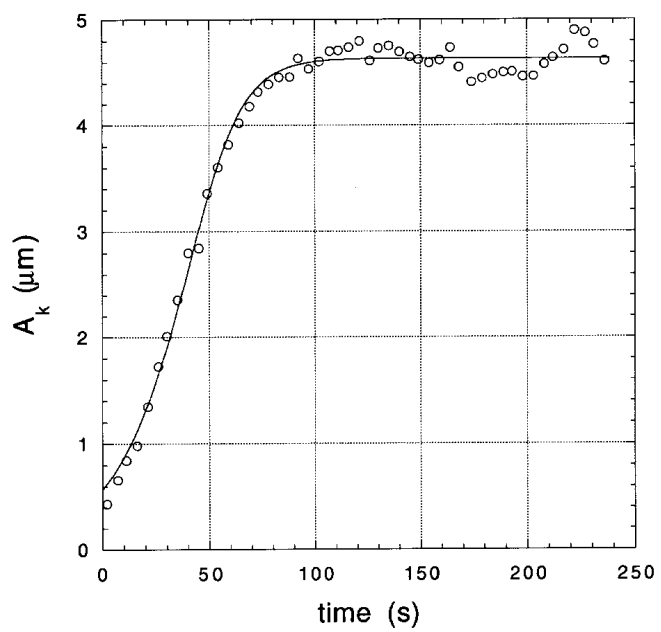

Figure 8. Circles are the measured amplitude of the most unstable spatial Fourier mode of the interface of Fig. 7, as a function of time. Continuous line is a fit using a solution of the third-order Landau amplitude equation given by Eq. $(20 . b)$.

For a particular temperature gradient, the planar nematic-isotropic interface becomes cellular above some critical velocity. An example of the growth of the cellular instability as a function of time is shown in Fig. 7. The amplitude of the most unstable spatial Fourier mode is displayed in Fig. 8. The data are the circles and the continuous curve is a fit to a solution of the third-order Landau amplitude equation given by Eq. 
(20.a). From the fitting we obtain the growth rate of the most unstable mode $\omega$ and $\sigma$ the third-order coefficient. In a previous article we showed that in the regime where a third-order Landau equation fits well the data, the results are consistent with the theory of Caroli et al. [10 ] for this instability. As we increase growth velocity, secondary instabilities start to appear and the dynamical behavior of the interface becomes very complex. An example of a secondary instability is shown in Fig. 9. This is an vacillating-breathing instability, where the interface shows a spatial period-doubling oscillatory instability. The cell width oscillates in phase opposition with its neighbors (vacillation) and we also notice that the cell top oscillates in a breathing fashion. These motions are quasi-periodic and eventually the interface becomes chaotic [14 ]. In Fig. 10 we show the time evolution of the main spatial Fourier mode of the patterns shown in Fig. 9. The oscillations are quasiperiodic and the system evolves to a scenario of spatiotemporal chaos.

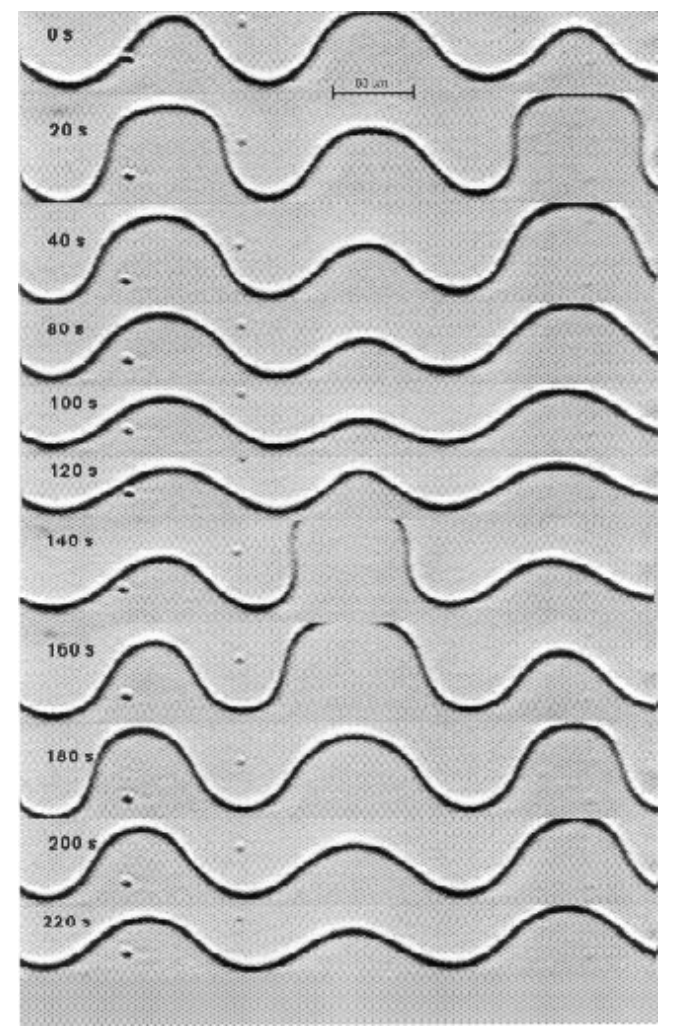

Figure 9. Time sequence of a moving nematic-isotropic interface undergoing a vacillating-breathing instability. Note that the cell width oscillates in phase opposition with its neighbors (vacillation) while the cell top oscillates in a breathing fashion. $t=0 \mathrm{~s}$ in this figure corresponds to $\mathrm{t}=1500 \mathrm{~s}$ in the actual experiment.

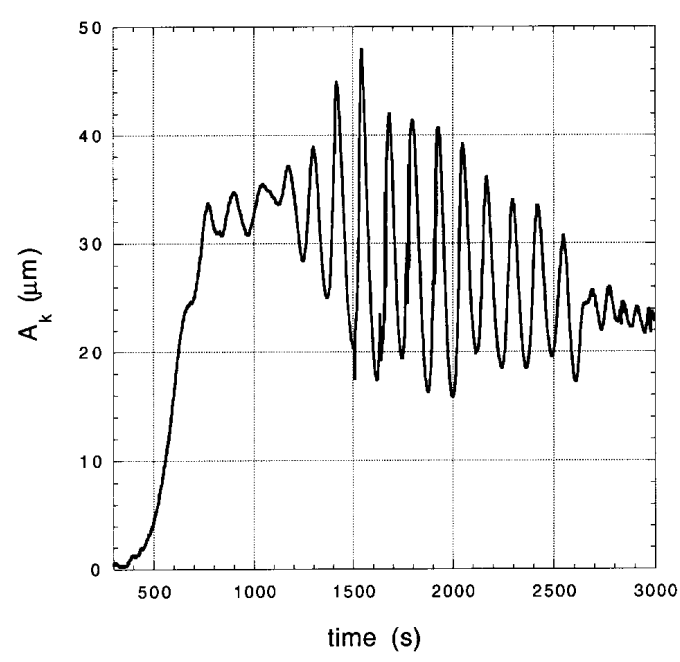

Figure 10. Amplitude of the most unstable spatial Fourier mode of the nematic-isotropic interface from Fig. 9, as a function of time. Pictures shown in Fig. 9 correspond to interface shapes recorded from $t=1500 \mathrm{~s}$, while the plot of the Fourier amplitude is for the whole run. We see a quasiperiodic motion due to the vacillating-breathing instability. Later on, the interface became chaotic.

More complex dynamical situations can be obtained and a more complete characterization of these secondary instabilities are under way in our laboratory.

\section{Conclusions}

Nonequilibrium nematic-isotropic interfaces are very convenient systems to study dynamics of first-order phase transitions. Since a Landau-de Gennes free energy description for this phase transition is available, predictions about the kinetics of growth can be made. We presented calculations on the kinetics of the nematic phase growing into a metastable isotropic phase and, for the first time, kinetic measurements which compare well with the theory presented. The nonequilibrium nematic-isotropic interface is also a very good model system to study morphological instabilities and pattern formation during growth of binary mixtures. Because the difference in free energy of the nematic and isotropic phases is small (weakly-first-order transition), segregation coefficients are of the order of 0.7 to 0.9 what makes experimentally easier and faster to work with liquid crystals as compared to regular crystal-melt interfaces. Also the latent heat produced during growth can be safely neglected what makes the analysis of the experimental results much simpler and more direct for 
comparison with theoretical models. We present some examples of the nematic-isotropic interfaces undergoing a cellular instability. Also we showed an example of a vacillating-breathing secondary instability which can drive the system to a scenario of spatio-temporal chaos. As the system becomes far from equilibrium, very complex dynamical behavior occurs. Liquid crystals offer new possibilities as compared to regular solid-fluid systems, because the degree of order in both phases can be varied under the action of electric fields. Since the applied electric field can be varied continuously, some parameters like segregation coefficient, capillary length and anisotropy of the surface tension may as well be varied continuously. Since this feature is unique for liquid crystals, this opens up new interesting possibilities for experiments. Interesting experiments would be on the cellular-dendritic transition and test of the theory of microscopic solvability for dendrites, both requiring a continuous variation of surface tension anisotropy.

In this paper we reviewed two different aspects of the moving nematic-isotropic interface problem. Hopefully, it will give to the readers a flavor about this area of research and about the wealth of phenomena and complex dynamical behavior that this conceptually simple system can display.

\section{Acknowledgments}

I would like to thank the members of our Statistical Physics Group specially Prof. J.M.A. Figueiredo, Prof. J.K.L. Silva and the students Orlando A. Gomes, Nathan B. Viana and Ubirajara A. Batista. Work in our laboratory has been sponsored by FAPEMIG and fellowships provided by $\mathrm{CNPq}$.

\section{References}

[1] Paul Manneville, Dissipative Structures and Weak Turbulence, Academic Press, New York, 1990.

[2 ] M.C. Cross and P.C. Hohenberg, Rev. Mod. Phys. 65, 851 (1993).

[3 ] Wim van Saarlos, Phys. Rev. A 37, 211 (1988).

[4] Wim van Saarlos, Phys. Rev. A 39, 6367 (1989).

[5 ] V. Popa-Nita and T.J. Sluckin, J.Phys. II France 6, 873 (1996).

[6 ] Nathan B. Viana, Master's Thesis, UFMG, Belo Horizonte, Brasil (1998).

[7] Nathan B. Viana, J.K.L. Silva, O.N. Mesquita and J.M.A. Figueiredo, submitted to publication (1998).

[8 ] W.W. Mullins and R.F. Sekerka, J. Appl. Phys. 34, 323 (1963) ; 35, 444 (1964).

[9 ] J.S. Langer, Rev. Mod. Phys. 52, 1 (1980), and references therein.

[10 ] B. Caroli, C. Caroli, and B. Roulet, J.Phys. (Paris) 43, 1767 (1982).

[11 ] P. Oswald, J. Bechhoefer, and A. Libchaber, Phys. Rev. Lett. 58, 2318 (1987).

[12 ] J.M.A. Figueiredo, M.B.L. Santos, L.O. Ladeira, and O.N. Mesquita, Phys. Rev. Lett. 71, 4397 (1993).

[13 ] J.M.A. Figueiredo and O.N. Mesquita, Phys. Rev. E 53, 2423 (1996).

[14 ] K. Kassner, C. Misbah, H. Muller-Krumbhaar, and A. Valance, Phys. Rev. E 49, 5477 and 5495 (1994).

[15 ] J.-M. Flesselles, A. Simon and A. Libchaber, Adv. Physics 40, 1 (1991).

[16 ] P.G. de Gennes and J. Prost, The Physics of Liquid Crystals, Claredon Press, Oxford (1993).

[17 ] S. Faetti and V. Palleschi, J. Chem. Phys. 81, 6254 (1984).

[18 ] P. Olmsted and P. Goldbart, Phys. Rev. A 46, 4966 (1992).

[19 ] E. Ben-Jacob, H.R. Brand, G. Dee, L. Kramer, and J.S. Langer, Physica D 14, 348 (1985).

[20 ] P. Coullet and G. Iooss, Phys. Rev. Lett. 64, 866 (1990).

[21 ] O.A. Gomes, J.M.A. Figueiredo and O.N. Mesquita, submitted to publication (1998). 